\title{
Gene editing enables T-cell engineering to redirect antigen specificity for potent tumor rejection
}

\author{
Julian J Albers ${ }^{1}$, Tim Ammon², Dario Gosmann¹, Stefan Audehm¹, Silvia Thoene ${ }^{3,4}$, Christof Winter ${ }^{3,4} \mathbb{D}$, Ramona Secci ${ }^{3}$, \\ Anja Wolf ${ }^{5}$, Anja Stelzl ${ }^{1}$, Katja Steiger ${ }^{6,4}$, Jürgen Ruland ${ }^{3,4,7}$, Florian Bassermann ${ }^{1,4}$, Christian Kupatt ${ }^{5,8}$, Martina Anton ${ }^{9}$, \\ Angela M Krackhardt1,4
}

\begin{abstract}
Adoptive transfer of TCR transgenic T cells holds great promise for treating various cancers. So far, mainly semi-randomly integrating vectors have been used to genetically modify $T$ cells. These carry the risk of insertional mutagenesis, and the sole addition of an exogenous TCR potentially results in the mispairing of TCR chains with endogenous ones. Established approaches using nonviral vectors, such as transposons, already reduce the risk of insertional mutagenesis but have not accomplished site-specific integration. Here, we used CRISPR-Cas9 RNPs and adeno-associated virus 6 for gene targeting to deliver an engineered TCR gene specifically to the TCR alpha constant locus, thus placing it under endogenous transcriptional control. Our data demonstrate that this approach replaces the endogenous TCR, functionally redirects the edited T cells' specificity in vitro, and facilitates potent tumor rejection in an in vivo xenograft model.
\end{abstract}

DOI 10.26508/lsa.201900367 | Received 5 March 2019 | Revised 6 March 2019 | Accepted 7 March 2019 | Published online 15 March 2019

\section{Introduction}

In recent years, the adoptive transfer of genetically reprogrammed T cells has gained more and more momentum (Lim and June 2017; June \& Sadelain, 2018). Although the use of T cells expressing a chimeric antigen receptor (CAR T cells) has already advanced to the clinic, the adoptive transfer of TCR transgenic cells (TCR T cells) is less far developed (Morgan et al, 2006; Rapoport et al, 2015; Tran et al, 2016). Nonetheless, TCR T cells hold promise for also targeting intracellular antigens and thereby greatly enlarge the scope of potentially targetable antigens (Harris \& Kranz, 2016), including neoantigens which are very attractive targets for personalized tumor therapy (Bassani-Sternberg et al, 2016; Stronen et al, 2016; Zacharakis et al, 2018).

A main challenge in engineering TCR $T$ cells is the genetic modification of primary $T$ cells. Previously, lenti-, $y$-retroviruses or nonviral vectors, such as transposons, were the vector of choice for stable integration of the tumor-reactive TCR (Morgan et al, 2006; Peng et al, 2009; Robbins et al, 2011; Rapoport et al, 2015; Rosenberg \& Restifo, 2015; Deniger et al, 2016; Clauss et al, 2018). Although integrating viral vectors are in clinical use, they carry the risk of insertional mutagenesis (Hacein-Bey-Abina et al, 2003). With the advent of individualized cellular immunotherapies that target patient-specific antigens, it is also essential to produce personalized vectors, which will further increase the effort needed for clinical translation (Krackhardt et al, 2018). Nonviral vectors such as transposons are easier to implement clinically and show a more favorable integration pattern, but also only integrate in a nonspecific fashion (Tipanee et al, 2017).

Another problem with these approaches is that they only add an additional TCR gene to the already existing, endogenous one instead of replacing it (Bendle et al, 2010; van Loenen et al, 2010). This poses a problem as the introduced TCR competes with the endogenous TCR for CD3-binding sites and thus for surface expression (Ahmadi et al, 2011). The introduced TCR chains could also potentially bind endogenous chains, leading to new TCRs with potentially hazardous specificities (Bendle et al, 2010; van Loenen et al, 2010). Several engineering strategies were developed to avoid these problems by increasing the specific binding of the introduced TCR chains and to improve surface expression of the introduced TCR. These include, for instances, codon optimization (Scholten et al, 2006), the usage of a self-cleaving $2 \mathrm{~A}$ peptide for equimolar expression (Leisegang et al, 2008), the exchange of the constant regions of the $\alpha$ - and $\beta$ chains for murine sequences (Cohen et al, 2006) and the introduction of additional cysteine bonds (Kuball

${ }^{1}$ Klinik und Poliklinik für Innere Medizin III, Klinikum Rechts der Isar, Technische Universität München, Munich, Germany ${ }^{2}$ Experimental Hematology Group, Klinik und Poliklinik für Innere Medizin III, Klinikum Rechts der Isar, Technische Universität München, Munich, Germany ${ }^{3}$ Institut für Klinische Chemie und Pathobiochemie, Klinikum Rechts der Isar, Technische Universität München, Munich, Germany ${ }^{4}$ German Cancer Consortium (DKTK), Partner-site Munich and German Cancer Research Center (DKFZ), Heidelberg, Germany ${ }^{5}$ Klinik und Poliklinik für Innere Medizin I, Klinikum Rechts der Isar, Technische Universität München, Munich, Germany ${ }^{6}$ Institut für Allgemeine Pathologie und Pathologische Anatomie, Technische Universität München, Munich, Germany ${ }^{7}$ German Center for Infection Research (DZIF), Partner Site Munich, Munich, Germany ${ }^{8}$ German Center for Cardiovascular Research (DZHK), Partner-Site Munich Heart Alliance, Munich, Germany ${ }^{9}$ Institut für Molekulare Immunologie und Experimentelle Onkologie und Therapieforschung, Klinikum Rechts der Isar, Technische Universität München, Munich, Germany 
et al, 2007). Additional strategies include swapping domains between the $\alpha$ - and $\beta$ chain, adding a leucine fusion protein, or using single-chain TCRs (Govers et al, 2010; Knies et al, 2016; Foley et al, 2017). Other approaches focus on the endogenous TCR and aim to reduce its expression by disrupting the TCR gene with nucleases (Provasi et al, 2012; Legut et al, 2018) or silencing it with miRNAs (Clauss et al, 2018).

CRISPR-Cas9 has been proven to be an efficient method for gene disruption in primary human T cells (Osborn et al, 2016; Knipping et al, 2017; Seki \& Rutz, 2018) and is already being used in clinical trials (NCT03399448 [ClinicalTrials.gov 2018]). In fact, targeted integration of a CAR into the TCR alpha constant (TRAC) locus was shown to potently redirect T cells against new antigens (Hale et al, 2017; MacLeod et al, 2017). Furthermore, placing the transcription of the transgene under the control of the endogenous promoter enhanced in vivo tumor control in mice (Eyquem et al, 2017). Just recently, it was demonstrated that co-electroporation of RNPs and double-strand DNA (dsDNA) enabled targeted integration of a shortened TCR construct into the TRAC locus. The introduced TCR construct lacks the constant region of the TCR $\alpha$ chain and uses the endogenous promoter and the endogenous $\alpha$ constant region when correctly integrated (Roth et al, 2018). Although this design elegantly illustrates the possibilities of targeted integration, it relies on the endogenous TRAC sequence and thus hinders TCR engineering strategies modifying this region of the introduced TCRs.

Here, we used CRISPR-Cas9 RNPs and adeno-associated viruses (AAV6) to site specifically integrate a $2.3-\mathrm{kb}$-long TCR construct into the TRAC locus, thereby replacing the endogenous TCR. By using a codon-optimized, complete TCR construct with murine constant regions and an additional disulfide bond, we were able to combine the advantages of engineered TCR constructs with those of the targeted integration of the transgene.

Our data show that targeting a TCR to the TRAC locus and placing it under the transcriptional control of the endogenous regulatory network redirects the specificity of the modified T cells and enables them to specifically eliminate tumor cells in vitro and in a murine in vivo tumor xenograft model.

\section{Results}

\section{Targeted integration of a TCR into the TRAC locus}

To induce a double-strand break in the gene encoding the TCR $\alpha$ chain, we designed a gRNA targeting the first exon of the TRAC locus. This region is specifically attractive as it is shared between all rearranged $T$ cells, and a disruption in the first exon is located upstream of the functional region needed for surface expression (Eyquem et al, 2017). CRISPR-Cas9 RNPs were used to induce the double-strand break as they were shown to be a highly efficient delivery method of CRISPR-Cas9 for primary human T cells (Schumann et al, 2015; Seki \& Rutz, 2018). Flow cytometric analysis of the cells showed an average knockout efficiency of 51\% (Fig 1A). The knockout was confirmed by Droplet Digital PCR (ddPCR) (Mock et al, 2016), which quantified the gene-editing frequency of TRAC alleles as 40\% using $10 \mathrm{ng}$ genomic DNA input (Fig 1B and C). Using $100 \mathrm{ng}$ genomic DNA input, the gene-editing frequency was $47 \%$, which is in line with the flow cytometric analysis (Fig S1).

Next, we designed a targeting construct to knock-in a TCR into the TRAC locus via HDR. For this, we used the previously described TCR2.5D6 (Klar et al, 2014). It was shown to recognize a myeloperoxidase-derived peptide, representing a tumorassociated antigen in patients with myeloid neoplasias, when presented on HLA-B7. The TCR construct was designed as a promoter trap to capture the endogenous promoter of the TRAC locus when it correctly integrates, thereby omitting the need for exogenous regulatory elements that risk insertional mutagenesis (Hacein-Bey-Abina et al, 2003). Furthermore, the TCR construct has a codon-optimized sequence, murine constant regions, and an additional disulfide bond and, thus, allows TCR engineering to enhance T-cell functionality. The TCR-targeting vector (Fig 1D) was delivered by AAV6, previously demonstrated to transduce human T cells (Eyquem et al, 2017; Hale et al, 2017; MacLeod et al, 2017). Edited cells expressed the exogenous TCR on their surface after RNP electroporation and subsequent AAV6 transduction, as determined by flow cytometry analysis (Fig 1E). The knock-in efficiency was on average 18\% (Fig 1F). This confirms that the correctly integrated transgene can be expressed without an exogenous promoter by using the endogenous one with a promoter trap construct.

To assess whether the introduced transgene was integrated into the TRAC locus as expected, genomic DNA was analyzed with ddPCR. For this, we designed two assays amplifying a region spanning either the whole left (LHA) or the whole right homology arm (RHA), with primer-binding sites in genomic regions and within the transgene. The targeted transgene integration efficiency was $27 \%$ (Fig $1 \mathrm{G}$ and $\mathrm{H}$ ).

Previous studies demonstrated that gene disruption of the endogenous TCR locus and subsequent lentiviral introduction of an exogenous TCR result in higher surface expression of the transgenic TCR and decreased off-target reactivity (Provasi et al, 2012). To clarify whether our knock-in approach also exchanged the surfaceexpressed TCR instead of just adding an additional TCR, thus creating double-positive T cells, we compared the double-positive rate of gene-edited T cells (KI-TRAC-TCR T cells) with that of retrovirally transduced cells (RV-TCR T cells). In KI-TRAC-TCR T cells, the portion of cells expressing both the endogenous human (TCRh) and the exogenous murinized TCR (TCRmu) was significantly decreased (Fig $1 E$ and I). Together, these data demonstrate that RNP electroporation and subsequent AAV6 transduction facilitate the targeted integration of the exogenous TCR in the TRAC locus, thus placing it under endogenous transcriptional control. Furthermore, the reduced double-positive rate in KI-TRAC-TCR cells strongly suggests that the knock-in of a TCR into the TRAC locus not only introduces the exogenous but also replaces the endogenous TCR.

\section{KI-TRAC-TCR T cells specifically recognize and lyse tumor cells in vitro}

We set out to assess whether the observed surface expression of the introduced TCR also specifically redirects the edited T cells and whether the editing process has a negative impact on functional capacities of redirected T cells. For this, the edited T cells were coincubated together with ML-2 acute myeloid leukemia (AML) cells that endogenously present the myeloperoxidase peptide, and which 
A

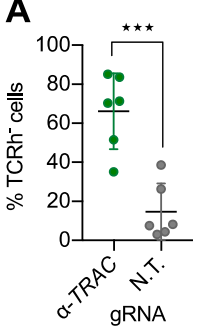

D

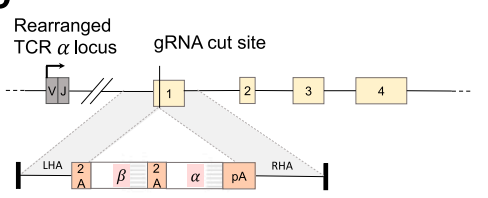

Edited TCR $\alpha$ locus

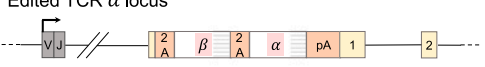

C
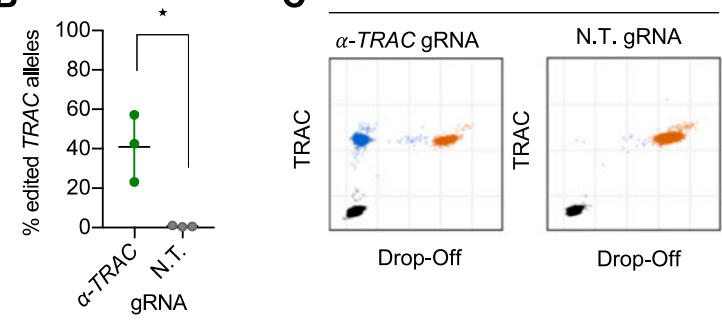

Drop-Off
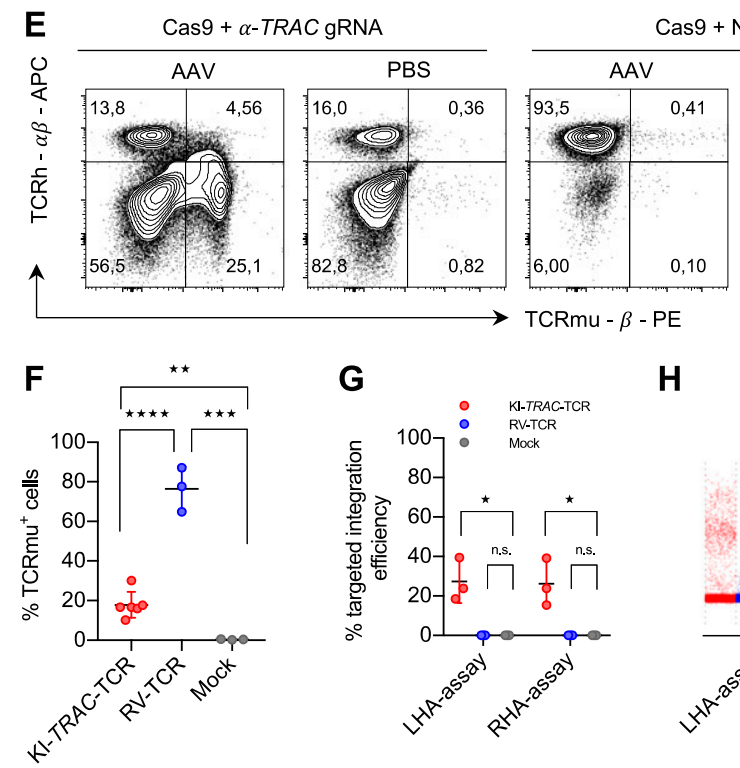

G

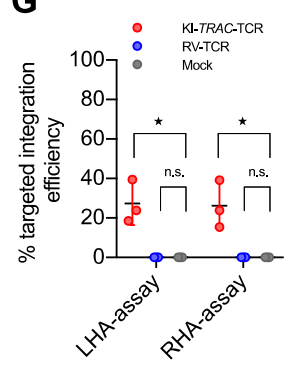

H

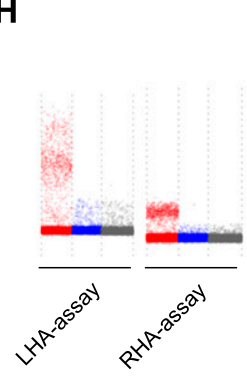

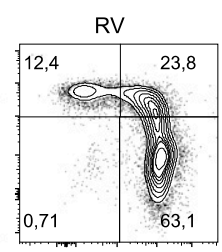

I

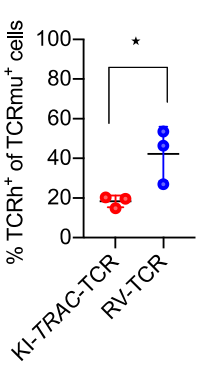

Figure 1. CRISPR-Cas9- and AAV-mediated TCR replacement.

(A) Flow cytometry analysis of primary human CD8 T cells electroporated with RNPs with an $\alpha$-TRAC gRNA or a non-targeting (N.T.) gRNA at day 7 after electroporation (data represent three donors in two independent experiments, $n=6$ ). (B) ddPCR quantification of the percentage of edited TRAC alleles on day 7 ( $n=3$ donors) with $10 \mathrm{ng}$ genomic DNA input. (C) Representative ddPCR plots are shown. $x$ and $y$ axes show fluorescence intensity (arbitrary units). (D) Schematic representation of the human TRAC locus (top), the recombinant AAV6 targeting construct encoding the exogenous TCR (middle) and the successfully edited TRAC locus (bottom). LHA, about 900-bp-long left homology arm; RHA, about 900-bp-long right homology arm. (E) Representative FACS plots of primary CD8 T cells electroporated with $\alpha-T R A C$ or N.T. gRNA and transduced with AAV $\left(\mathrm{MOI}=10^{6}\right)$ or PBS or $\gamma$-retrovirally transduced on day 7 after electroporation or transduction. Axes use biexponential scaling. Graphs are $10 \%$ contour plots with outliers displayed. (F) Flow cytometry analysis of KI-TRAC-TCR cells (data represent three donors in two independent experiments, $n=6$ ), $y$-retrovirally ( $n=3$ donors), or mock-transduced cells ( $n=3$ donors). (G) ddPCR quantification of the targeted integration efficiency with assays spanning the left (LHA-assay) or right homology arm (RHA-assay). (H) Representative ddPCR plots are shown. y axis shows fluorescence intensity (arbitrary units). (I, F) Flow cytometry analysis as in (F) ( $n=3$ donors). Asterisks indicate statistical significance as determined by twotailed unpaired $t$ test. See also Fig S1. were transgenic for HLA-B7 or control HLA-B15 (Mall et al, 2016). KITRAC-TCR T cells lysed the tumor cells expressing HLA-B7, whereas not lysing the HLA-B15-bearing control cells (Fig 2A). This demonstrates that the targeted integration of a TCR in the TRAC locus specifically redirects $T$ cells against a defined antigen and that the edited $T$ cells are able to lyse recognized tumor cells in vitro.

Whereas the portion of TCRmu-expressing cells was significantly higher in RV-TCR cells and retrovirally transduced TRAC-knockout T cells (RV-TCR TCRendo ${ }^{-}$T cells) compared with TCR knock-in cells, all modified T cells lysed target cells equally well in vitro at different $T$ cell to tumor ratios, after adjustment of effector to target ratios according to $\mathrm{TCRmu}^{+}$cells (Figs $2 \mathrm{~B}$ and $1 \mathrm{E}$ ). The differently genetically modified $\mathrm{T}$ cells also showed no marked difference in IFN- $\gamma$ secretion (Fig 2C). These findings demonstrate that our approach functionally redirects $T$ cells against defined antigens. During the editing procedure, $T$ cells maintain their ability to lyse target cells and secrete IFN- $\gamma$.

\section{KI-TRAC-TCR T cells potently reject xenograft tumors in vivo}

To explore whether edited T cells also defeat cancer cells in vivo, they were adoptively transferred into NSG mice bearing the subcutaneous AML xenograft ML-2 tumors. Over $3 \mathrm{~d}$, a total of $2 \times 10^{7} \mathrm{TCRmu}{ }^{+} \mathrm{KI}-$ TRAC-TCR T cells were adoptively transferred, resulting in a significant decrease of tumor size (Fig 3A). When the experiment was terminated on day 7 after the first T-cell dose, most tumors were no longer macroscopically detectable. The tissue at the tumor injection site was harvested, and upon histological examination, only small tumor remnants were visible (Fig 3B). Immunohistochemistry staining for CD3 revealed a massive infiltration of human $T$ cells in the tumor remnants (Fig 3C). When compared with mice treated with RV-TCR $T$ cells or RV-TCR TCRendo- $T$ cells, no notable differences were observed (Figs 3A, D-G and S2). In mice treated only with mocktransduced $T$ cells, on the other hand, we detected large tumors without T-cell infiltration (Fig $3 \mathrm{~A}, \mathrm{H}-\mathrm{K}$ ). These findings demonstrate 
A

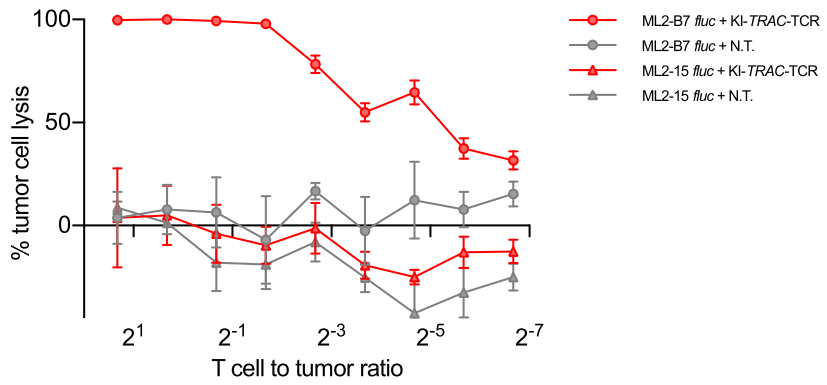

B

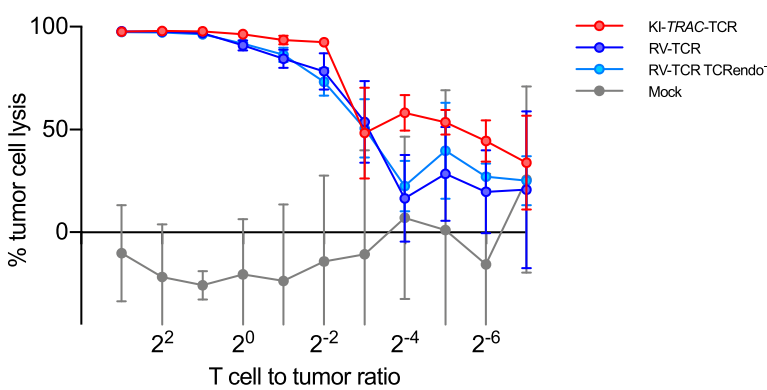

C

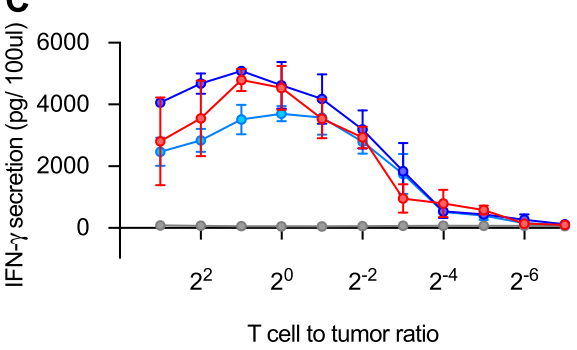

Figure 2. Targeted integration of a TCR into the TRAC locus redirects T-cell specificity.

(A) Cytotoxic lysis of firefly luciferase (fluc)-expressing ML2 cells expressing HLAB7 or HLA-B15 by KI-TRAC-TCR cells or unedited T cells at indicated T cell to tumor cell ratios after $24 \mathrm{~h}$ of co-incubation assessed by luminescence $(n=3$ technical replicates of 1 donor). (B) fluc cytotoxicity assay comparing KI-TRAC-TCR cells, RV-TCR, RV-TCR TCRendo-, and mock cells after $24 \mathrm{~h}$ of co-incubation with ML2-B7 fluc at indicated T cell to tumor ratios. T cell to tumor ratios were calculated based on the portion of TCRmu ${ }^{+}$cells, and equal numbers of TCRmu ${ }^{+}$ cells of each condition were used for co-incubation ( $n=3$ technical replicates of two donors). (C) IFN- $y$ ELISA of the supernatant.

that redirected $T$ cells function very effectively within an in vivo mouse model. The $T$ cells were able to migrate and home to the tumor, lyse the tumor cells within the tumor microenvironment, and reject the tumor in this mouse model of AML within $7 \mathrm{~d}$.

\section{Discussion}

Here, we demonstrate that the specificity of T cells can be redirected by targeting an engineered TCR to the TRAC locus using CRISPR-Cas9 RNPS and AAV6 to functionally replace the endogenous TCR with an exogenous, tumor-reactive TCR. The edited cells specifically lyse tumor cells in vitro and control tumors in vivo in a xenograft model of AML.

The observed rate of transgene expression is higher than the previously reported knock-in rate for a TCR into the TRAC locus using dsDNA as a donor template (Roth et al, 2018), even though the introduced TCR construct was 2.3-kb long and contained the complete $\alpha$ - and $\beta$ chains, therefore permitting TCR engineering approaches to modify the entire TCR $\alpha$ gene. This emphasizes the attractivity of AAV6 as donor templates for HDR-dependent integration. The specific integration of the transgene into the intended locus is consistent with the targeted locus amplificationsequencing profiles presented by previous studies, when targeting transgenes to the TRAC locus (Eyquem et al, 2017; Roth et al, 2018). These data confirm that HDR is a reliable mechanism to specifically introduce defined TCR transgenes into the TRAC locus. Thus, this approach is suitable to reduce the risk of nonspecific integration in a different locus which could transform edited cells. The use of AAV6 as an HDR donor template is especially attractive as its genome is composed of single-strand DNA. Previous studies have demonstrated that single-strand DNA donor templates integrate more specifically at the intended target site and, therefore may enhance safety of this approach in comparison with doublestrand DNA donor templates, which also integrate in an HDRindependent manner at other sites of double-strand breaks (Murnane et al, 1990; Suzuki et al, 2016; Roth et al, 2018). This is important because CRISPR-Cas9 is known to also induce off-target double-strand breaks, into which a transgene could aberrantly integrate (Zhang et al, 2015). Nonetheless, even if there is a small percentage of off-target integration, our HDR-dependent integration approach demonstrates greatly enhanced specificity compared with semi-randomly integrating vectors.

The targeted integration resulted in a significantly reduced proportion of cells expressing the endogenous TCR compared with randomly integrated TCR genes using a retrovirus for genetic transfer. Therefore, the transfer of T cells expressing mixed TCRs can be significantly reduced while providing equal efficacy in vitro as well as in vivo when targeted integration is used instead of retroviral transduction, as shown by our experiments with equilibrated numbers of TCRmu ${ }^{+}$cells. Thus, this technique provides an important safety advantage compared with other non-targeted gene transfer approaches using viruses or transposons, given that expression of mixed TCR may result in potential harmful toxicities as described in an animal model (Bendle et al, 2010).

Previous studies placing a tumor-reactive receptor under transcriptional control of the TRAC locus used xenograft mouse models in which tumor burden was reduced by adoptive T-cell transfer, but the tumors could not be fully removed. In this setting, they were able to demonstrate that edited T cells using the endogenous transcriptional regulation showed better antitumor activity, and in the case of CAR knock-in, a significantly prolonged median survival compared with retrovirally transduced T cells (Eyquem et al, 2017; Roth et al, 2018). In our experiments, we were able to show a strong and fast tumor rejection potential by redirected KI-TRAC-TCR T cells, albeit this was not superior in comparison with RV-TCR T cells or RV-TCR TCRendo ${ }^{-} \mathrm{T}$ cells. This could be attributed to the fact that we adoptively transferred a higher number of TCR T cells and used a tumor rejection model, where $T$ cells eliminated the tumor within a short period of time. Thus, T-cell exhaustion may not play a major role in our model compared with the models used in previous publications (Eyquem et al, 2017; Roth et al, 2018). Nevertheless, KI-TRAC-TCR T cells 
䖪路 Life Science Alliance
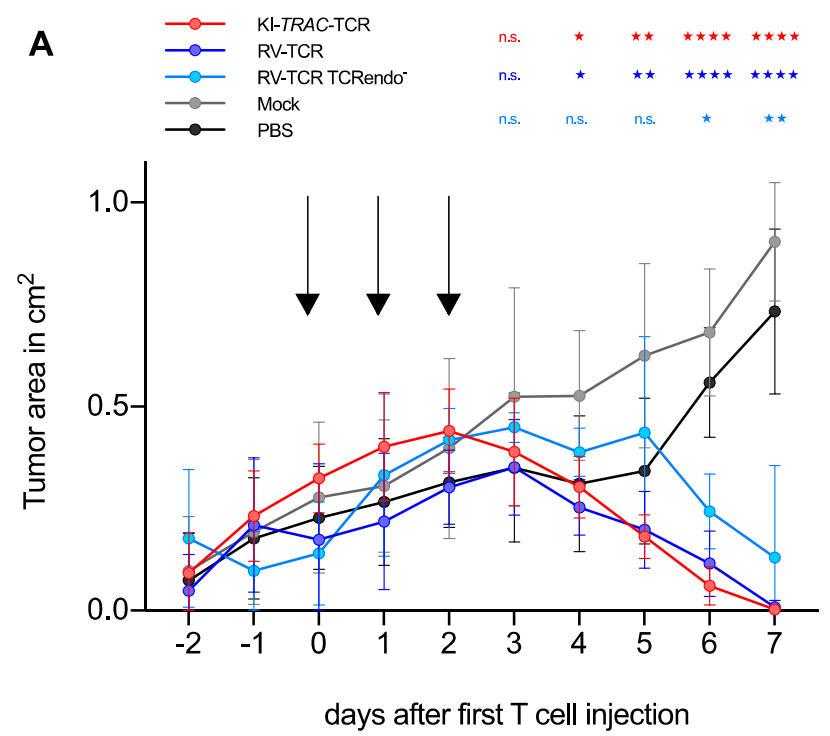

B

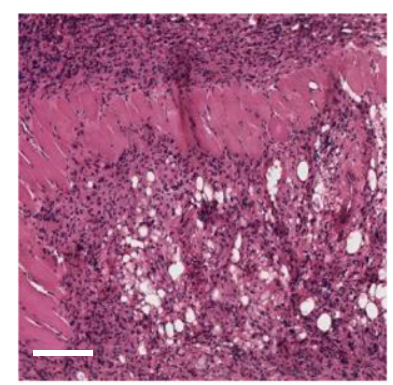

$\mathrm{KI}$-TRAC-TCR - HE

D

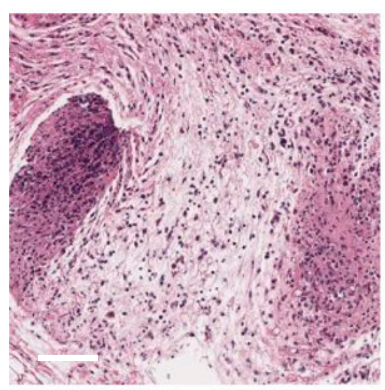

RV-TCR - HE

$\mathbf{F}$

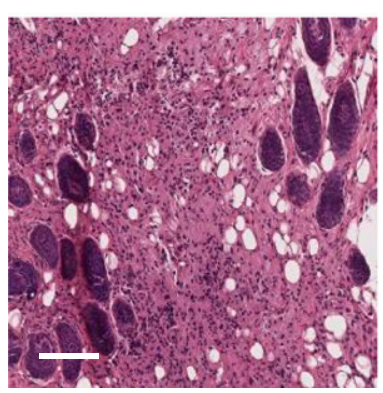

RV-TCR TCRendo- - HE
C

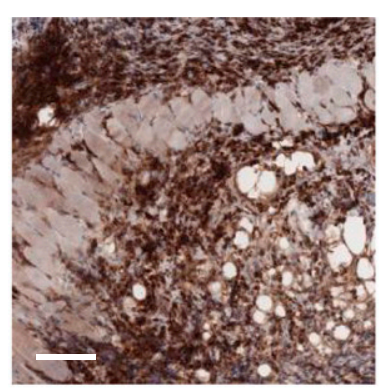

KI-TRAC-TCR - CD3

E

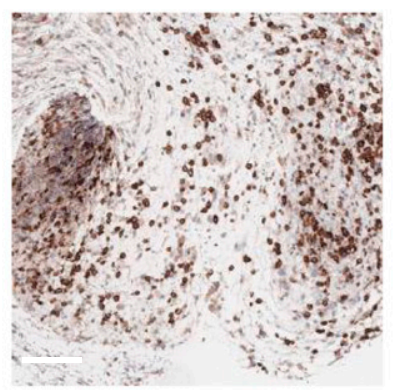

RV-TCR - CD3

G

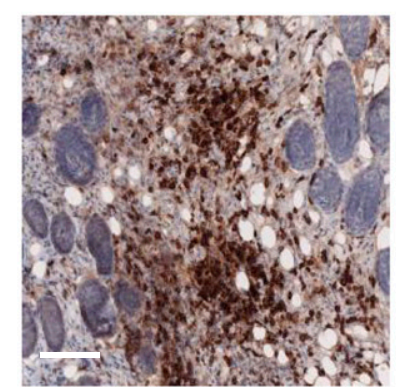

RV-TCR TCRendo- - CD3

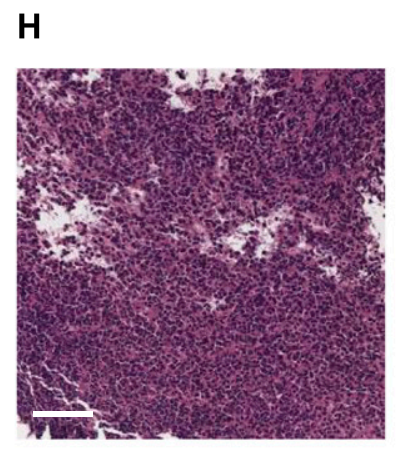

I

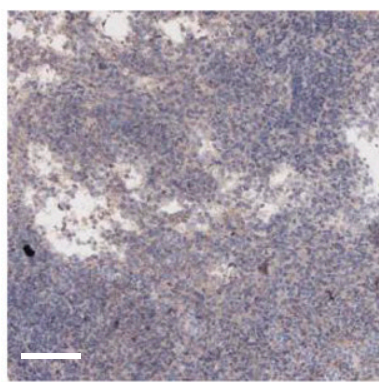

Mock - HE

J

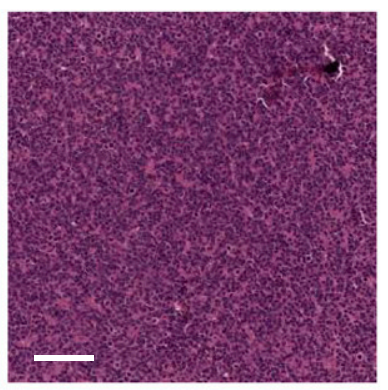

PBS - HE
Mock - CD3

K

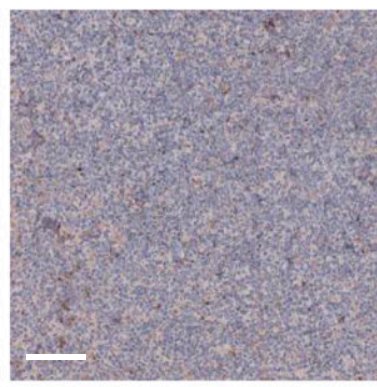

PBS - CD3 
showed non-inferiority harboring all the advantages of specific integration. Our approach provides a base for in-depth comparisons with $T$ cells modified by targeted integration using dsDNA as well as non-targeted integration by viruses and transposons with respect to subtle differences in safety as well as short- and longterm antitumor immunity.

The method described here can easily be implemented on a laboratory scale and enables researchers to further investigate TCR biology. The role of the regulatory transcriptional network on T-cell functionality and exhaustion in particular can further be addressed. In addition, the targeted integration of TCRs could potentially be adopted for clinical translation as both elements-CRISPR-Cas9 and adeno-associated viruses-are already applied in clinical trials or are even clinically approved. This could lead the field of adoptive T-cell transfer away from randomly integrating vectors and pave the way for new cellular therapies.

\section{Materials and Methods}

\section{gRNA}

The gRNA targeting the first exon of the TRAC locus was designed with the WTSI Genome Editing tool (Hodgkins et al, 2015). The nontargeting gRNA was previously described (Doench et al, 2014). Both RNAs were synthesized by Integrated DNA Technologies as crRNAs and subsequently duplexed with tracrRNAs. $\alpha$-TRAC gRNA target sequence: $5^{\prime}$-TCTCTCAGCTGGTACACGGC-3'; non-targeting gRNA: 5'-GTATTACTGATATTGGTGGG-3'.

\section{RNP production}

CRISRP-Cas9 RNPs were assembled from the crRNA:tracrRNA duplexes and Alt-R Sp Cas9 Nuclease (Integrated DNA Technologies) according to the manufacturer's recommendations. Briefly, crRNA and tracrRNA were equimolarly mixed and resuspended in IDTE buffer at a concentration of $44 \mu \mathrm{M}$. This mix was heated to $95^{\circ} \mathrm{C}$ for 5 min and let cool down to room temperature. crRNA:tracrRNA duplexes were mixed with $36 \mu \mathrm{M}$ Cas9 enzyme and incubated at room temperature for $20 \mathrm{~min}$. RNPs were prepared on the day of electroporation and stored at $4^{\circ} \mathrm{C}$.

\section{Isolation of primary human CD8 T cells for gene targeting}

Blood from healthy donors was acquired with informed consent according to the Helsinki Declaration and the local ethical board. PBMCs were isolated by density-gradient centrifugation with Ficoll/ Hypaque (Biochrom) and frozen in FCS supplemented with $10 \%$ DMSO. For genetic modification, the cells were thawed and CD8
T cells were isolated via negative selection with the Dynabeads Untouched Human CD8 T Cells kit (Thermo Fisher Scientific).

\section{Primary human T-cell culture and ML2 cell line}

T cells were kept at a density of $\sim 10^{6}$ cells per $\mathrm{ml}$ cell culture medium. The T-cell medium consisted of RPMI 1640 (Invitrogen), 1× penincillin/streptomycin (Invitrogen), 5\% FCS, 5\% human serum, $1 \mathrm{mM}$ sodium pyruvate (Invitrogen), $2 \mathrm{mM} \mathrm{L-glutamine} \mathrm{(Invitrogen),}$ $10 \mathrm{mM}$ nonessential amino acids (Invitrogen), $10 \mathrm{mM}$ Hepes (Invitrogen), and $16 \mu \mathrm{g} / \mathrm{ml}$ gentamycin (Biochrom). Human IL-7 and human IL-15 (both PeproTech) were added to the medium to a final concentration of $5 \mathrm{ng} / \mathrm{ml}$ each and replenished when fresh culture medium was added to the cells every 2-3 d. The AML cell line ML2 (The CABRI consortium) was retrovirally transduced with genes encoding firefly luciferase (fluc) or HLA-B7. Mycoplasma contamination status was regularly tested.

\section{Target vector construction}

The target vector PAAV-L900-PT-TCR2.5D6omc-R900 was cloned based on the plasmid PAAV-CMV-eGFP. The construct bears two inverted terminal repeats (ITRs) based on AAV2, which are cis-acting sequences needed for successful viral packaging of the sequences between the ITRs. Primers were designed to amplify homology arms covering 900-bp upstream or downstream of the intended double-strand break that has been induced by CRISPR-Cas9. The primers were designed in such a way that the protospacer adjacent motif was removed to prevent additional recognition by Cas9, and restriction enzyme-binding sites were added for subsequent restriction-ligation cloning. The homology arms were amplified from genomic DNA and cloned in pCR-Blunt plasmids (Thermo Fisher Scientific) and afterwards cloned between the ITRs of PAAV-CMV-GFP. The TCR construct used for the targeted integration in the TRAC locus was designed based on the previously described sequence of TCR2.5D6 (Klar et al, 2014). Briefly, in this sequence, the constant regions of the TCR $\alpha$ - and $\beta$ chain are replaced by murine sequences in which an additional cysteine bond is introduced. The $\alpha$ - and $\beta$ chains are connected by a P2A element to permit bicistronic expression from one promoter. To place the integrated TCR under the transcriptional control of the endogenous promoter of the TCR $\alpha$ chain, a P2A element was placed upstream of the TCR construct (Eyquem et al, 2017). This promoter trap was designed in such a way that it is located in frame within the first exon of the TRAC locus after successful integration. The construct was synthesized as GeneArt Strings DNA Fragment (Invitrogen). Subsequently, the integration cassette was cloned between the homology arms in the PAAV plasmid. The following primers were used for the amplification of the homology

Figure 3. T cells potently reject tumors in vivo independent of the way of genetic modification applied.

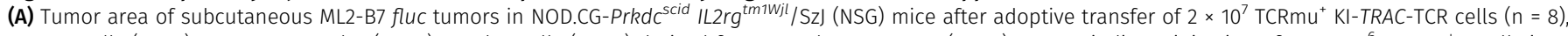
RV-TCR cells $(n=7)$, RV-TCR TCRendo $(n=3)$, mock T cells $(n=6)$ derived from one donor, or PBS $(n=6)$. Arrows indicate injection of $6.67 \times 10^{6}$ TCRmu ${ }^{+}$T cells in $200 \mu \mathrm{l}$ PBS. Asterisks indicate statistical significance as determined by multiple $t$ tests corrected for multiple comparisons using the Holm-Sidak method. (B-K) Representative HE and CD3 stains of tissue derived from mice treated with KI-TRAC-TCR cells (B, C), RV-TCR cells (D, E), RV-TCR TCRendo- (F, G), mock T cells (H, I), or PBS (J, K). Scale bars, $100 \mu \mathrm{m}$. See also Fig S2. 
arms: LHA_for: 5'-GGCGCGCCCACTAAGGAAAAG-3'; LHA_rev: 5' ACTAGTGTCAGGGTTCTGGATATCTG-3'; RHA_for: 5'-GGTAACCGTATACCAGCTTCGAGACTCTAAATCCAGTGACA-3'; RHA_rev: 5'-CGGTCCGCAAGTAGCATTTCTTCAGAG-3'.

\section{Recombinant AAV6 production}

Recombinant adeno-associated viruses of the serotype 6 were produced with the triple transfection method as described previously (Ziegler et al., 2017). Briefly, the packaging cell line U293 was transfected with the vector pAAV-L900-PT-TCR2.5D6omc-R900, a plasmid encoding the cap sequences of AAV6 and rep AAV2 sequences and the helper plasmid $\delta$ F6 (Puresyn) using PEI Max (Polysciences). After $72 \mathrm{~h}$, the cells were harvested and the virus was purified by iodixanol-gradient centrifugation.

The virus was further purified by a gravity flow size exclusion purification using Sepharose G100 SF resin (Sigma-Aldrich) in Econopac columns (Bio-Rad Laboratories) using the protocol kindly provided by the Salk Institute for Biological Studies.

The virus was concentrated in DPBS using Amicon Ultra-15 Centrifugal Filter Units (Merck) and stored at $4^{\circ} \mathrm{C}$. Viral titer was quantified by quantitative PCR as described previously (Aurnhammer et al, 2012).

\section{Gene targeting}

Primary human $T$ cells were activated with Dynabeads Human T-Activator CD3/CD28 (Thermo Fisher Scientific) according to the manufacturer's recommendations at a bead-to-cell ratio of 1:1 in the presence of $30 \mathrm{U} / \mathrm{ml}$ human $\mathrm{IL}-2$ (PeproTech) for $72 \mathrm{~h}$.

After bead removal, $10^{6}$ cells were washed in DPBS and resuspended in $11 \mu \mathrm{l}$ resuspension buffer $\mathrm{R}$ of the Neon electroporation kit (Invitrogen). $1 \mu \mathrm{l}$ of the RNP mix was added to the cells immediately before electroporation. $10 \mu \mathrm{l}$ of the mixture was electroporated with the Neon electroporation device at 1,600 V, $10 \mathrm{~s}$ with three pulses. The cells were transferred to $1 \mathrm{ml}$ of prewarmed T-cell medium. Immediately afterwards, the virus solution was added at a multiplicity of infection (MOI) of $10^{6}$ viral genomes per cell. For controls (TCRendo ${ }^{-}$T cells), the same volume of DPBS was added.

\section{$\gamma$-Retroviral transduction of $\mathrm{T}$ cells}

Primary human T cells were transduced with the pMP71-TCR2.5D6 construct previously described (Klar et al, 2014) according to a protocol previously published (Liang et al, 2010). The embryonal kidney cell line 293Vec-RD114 (BioVec Pharma) was used as the packaging cell line.

\section{ddPCR}

Genomic DNA was isolated from the cells with the DNeasy Blood \& Tissue kit (QIAGEN), following the manufacturer's instructions. DNA was quantified using a Qubit 2.0 fluorometer (Thermo Fisher Scientific). ddPCR analysis was performed on a QX200 ddPCR system with automatic droplet generation (Bio-Rad Laboratories). Reactions were carried out in ddPCR 96-well plates (\#12001925; BioRad Laboratories) and were performed in duplicate in a reaction volume of $21 \mu \mathrm{l}$ using the ddPCR Supermix for probes (no UTP,
Bio-Rad Laboratories) and with a template input of $10 \mathrm{ng}$ (LHA/RHA and Drop-Off/TRAC) or 100 ng DNA (EIF2C1 and Drop-Off/TRAC) per reaction. The locked nucleic acid probe and the EIF2C1 primers and probe were synthesized by Integrated DNA Technologies, and all other primers and hydrolysis probes were synthesized by SigmaAldrich: DO-TRAC-for: 5'-CTTGTCCATCACTGGCATCT-3'; DO-TRAC-rev: 5'-CGGTGAATAGGCAGACAGAC-3'; TRAC-probe: 5'-[6FAM]AGCCTGGGTTGGGGCAAAGAGGG[BHQ1] -3', LNA-Drop-Off: 5'-/5HEX/CCCTGC+C+ G+T+GTA/3IABKFQ/-3', LHA-for: 5'-CCCCAACATGCTAATCCTCC-3'; LHA-rev: 5'-ACAGCAGTCCCAGAGACATA-3'; LHA-probe: 5'-[6FAM]CCCCACAGAGCCCCGCCCT[BHQ1]-3', RHA-for: 5'-TCAGGTGATCTACCCACCTT-3'; RHA-rev: 5'-GTCTACCCTCTCATGGCCTA-3'; RHA-probe: 5'[HEX]CAGGGCCGGGTCACAGGGCC[BHQ1]-3', and EIF2C1-for: 5'-CCTGCCATGTGGAAGATGAT-3'; EIF2C1-rev: 5'-GAGTGTGGTCACTGGACTTG3'; EIF2C1-probe: 5' - /5HEX/ACCAGTCTG/ZEN/TGCGCCCTGCCA/ $3 / \mathrm{ABKFQ} /-3^{\prime}$. Primers were used at a final concentration of $900 \mathrm{nM}$ and probes at $250 \mathrm{nM}$. Genomic DNA derived from leukocytes of healthy subjects was used as negative control, Sanger-sequenced plasmid DNA as positive control, and purified, nuclease-free water as no-template control in all ddPCR runs. The thermal cycling protocol was as follows: $10 \mathrm{~min}$ at $95^{\circ} \mathrm{C}, 40$ cycles of $30 \mathrm{~s}$ at $94^{\circ} \mathrm{C}$, $1 \mathrm{~min}$ at $60^{\circ} \mathrm{C}$ for annealing/extension, and one final step of $10 \mathrm{~min}$ at $98^{\circ} \mathrm{C}$. For the LHA/RHA PCR, resulting in an amplicon of 2,337 bp, annealing/extension time was increased to $3 \mathrm{~min}$. In-PCR digestion was performed with HindIII (LHA/RHA), Haelll (EIF2C1), or Msel (Drop-Off/TRAC) in all cases. The plates were read on a QX200 droplet reader (Bio-Rad Laboratories) to determine droplet fluorescence intensity.

\section{ddPCR data analysis}

Droplets were manually assigned to double-negative, single - positive, or double-positive droplet clusters after visual inspection in QuantaSoft v1.7.4 (Bio-Rad Laboratories). Raw droplet fluorescence intensity values including cluster assignments were exported from QuantaSoft. Custom scripts were used to import the intensity values into $\mathrm{R}$ (version 3.4.4; http:/ / www.r-project.org), to generate plots, and to quantify sample concentrations. Target concentrations were calculated for each well from the number of positive droplets $\mathrm{Np}$ and negative droplets $\mathrm{Nn}$ and the average droplet volume $V=0.85 \mathrm{nl}$ based on Poisson distribution statistics using the formula $c=(\ln (N p+N n)-\ln (N n)) / V$, where $\ln$ is the natural logarithm. Following Mock et al (2016), rain droplets were considered negative because they represent mutated alleles. Gene-editing frequencies were calculated as the ratio of edited over edited plus wild-type allele concentrations.

\section{In vitro functional assays}

T cells were incubated together with $10^{4}$ firefly luciferase expressing ML-2 HLA-B7 fluc or ML-2 HLA-B15 fluc cells at indicated ratios in $200 \mu$ l cytokine-free T-cell medium. T cell to tumor ratios were calculated based on the portion of $\mathrm{TCRmu}^{+}$cells and equal numbers of $\mathrm{TCRmu}^{+}$cells of each condition were used for coincubation. After $24 \mathrm{~h}$, IFN- $y$ secretion into the supernatant was analyzed by ELISA (BD) according to the manufacturer's instructions. Cytotoxicity was assessed by adding $100 \mu \mathrm{l}$ luciferase 
substrate solution consisting of DPBS (Thermo Fisher Scientific), $10 \% \mathrm{FCS}$, and $150 \mu \mathrm{g} / \mathrm{ml}$ D-luciferin (Gold Biotechnology) to the cell pellet. Immediately afterwards, luminescence was determined with a VICTOR Multilabel Plate Reader (PerkinElmer). Tumor cell lysis was determined as tumor lysis $=(1-$ (luminescence of sample $)$ luminescence of untreated tumor cells) $\times 100)$. Negative lysis indicates cell growth compared with the untreated tumor cell controls.

\section{In vivo mouse model}

NOD.CG-Prkdc $c^{\text {scid }}$ IL2rg ${ }^{\text {tmiwjl }} /$ SzJ (NSG; The Jackson Laboratory) were maintained according to the institutional guidelines and approval of the local authorities. 107 ML2-B7 fluc cells were subcutaneously injected in the right flank of 8- to 12-wk-old mice. On day 8 after tumor cell injection, the mice were randomly assigned to the different treatment groups. On days 8,9 , and 10, edited T cells, retrovirally transduced $T$ cells, retrovirally transduced TCR knockout T cell, mock-transduced T cells, or PBS were intravenously injected. A total of $2 \times 10^{7} \mathrm{TCRmu}^{+} \mathrm{T}$ cells were injected in three doses of $200 \mu \mathrm{l}$ PBS each. As the transduction efficiency of the retrovirally treated cells was higher than that of the edited cells, mock-transduced cells were used to adjust the percentage of TCRmu ${ }^{+}$T cells in the treatment groups ( $\approx 20 \%)$. Tumors were measured daily with a caliper, and tumor area was calculated as $\mathrm{A}=$ length $\times$ width

\section{Flow cytometry}

The cells were stained with combinations of the following antibodies: anti-human TCR $\alpha / \beta$-PE (BW242/412; Miltenyi Biotec), antimouse TCR $\beta$ chain-APC (H57-597; BD), and anti-hCD3-PE (UCHT1; BD). In brief, 100,000 cells were washed in FACS buffer (PBS $+10 \%$ FCS) and blocked with $50 \mu$ of human serum for 10 min on ice. The cells were washed again with FACS buffer, stained with the respective antibodies, and incubated for 20 min on ice. Subsequently, the cells were washed with FACS buffer and fixed in 1\% PFA. The cells were analyzed with an LSRII Flow Cytometer (BD) or a CytoFLEX $\mathrm{S}$ (Beckman Coulter), and data were analyzed with Flowjo 10 (Flow)o LLC).

\section{Histology and immunohistochemistry}

Directly after euthanasia of the mice, tissues were fixed in $4 \%$ neutral-buffered formalin solution for at least $48 \mathrm{~h}$, dehydrated under standard conditions (ASP300S; Leica Biosystems) and embedded in paraffin. Serial $2-\mu \mathrm{m}$-thin sections prepared with a rotary microtome (HM355S; Thermo Fisher Scientific) were collected and subjected to histological and immunohistochemical analysis. Hematoxylin-eosin staining was performed on deparaffinized sections.

Immunohistochemistry was performed with a BenchMark XT automated stainer (Ventana) with an antibody against CD3 (103R-95, MRQ-39), using the ultraVIEW DAB Detection kit (all reagents were from Ventana). Briefly, the tissue sections were deparaffinized with EZ Prep at $75^{\circ} \mathrm{C}$ and $76^{\circ} \mathrm{C}$, heat-pretreated in Cell Conditioning 1 (CC1) solution for antigen retrieval at $76^{\circ} \mathrm{C}-100^{\circ} \mathrm{C}$ and then incubated with the primary antibody diluted in antibody diluent 1:500 for $32 \mathrm{~min}$ at $37^{\circ} \mathrm{C}$ after inactivation of the endogenous peroxidase using a UV inhibitor for $4 \mathrm{~min}$ at $37^{\circ} \mathrm{C}$. The slides were incubated with an HRP Universal Multimer for 8 min. Antibody binding was detected using DAB as chromogen and counterstained with hematoxylin for 10 min with subsequent bluing in bluing reagent for 10 min. The slides were then manually dehydrated by alcohol washes of increasing concentrations (70\%, 96\%, and 100\%) and xylene and cover-slipped using Pertex mounting medium (00801; Histolab). The stained slides were evaluated by an experienced certified pathologist (K Steiger) using a BX53 stereomicroscope (Olympus) and scanned using a slide scanner (AT-2; Leica Biosystems). Representative images were collected using Aperio Imagescope software (version 12.3; Leica Biosystems).

\section{Statistics}

All statistical analyses were performed as indicated in the figure legends using GraphPad Prism 7. Error bars in all figures represent mean \pm SD. ${ }^{*} P=0.01-0.05,{ }^{* *} P=0.001-0.01,{ }^{* *} P=0.0001-0.001$, and $\star \star \star \star * P<0.0001$.

\section{Supplementary Information}

Supplementary Information is available at https://doi.org/10.26508/lsa. 201900367.

\section{Acknowledgements}

We thank Roland Rad (TUM), Rupert Öllinger (TUM), Marc Schmidt-Supprian (TUM), Dieter Saur (TUM), and Roland Schmid (TUM) for their helpful discussion and comments. We thank all lab members for their valuable support. This research was supported by the Deutsche Forschungsgemeinschaft (SFB824/C10 to AM Krackhardt; SFB824/Z02 and SFB1335/Z01 to K Steiger and SFB 1054/B01, SFB 1335/P01 and P08, TRR 237/A10 to J Ruland), the European Research Council (FP7, grant agreement no. 322865) to J Ruland, and the TUM Medical Graduate Center to JJ Albers.

\section{Author Contributions}

J) Albers: conceptualization, data curation, formal analysis, investigation, visualization, methodology, and writing-original draft. T Ammon: supervision and writing-review and editing. D Gosmann: investigation and writing-review and editing.

S Audehm: investigation and writing-review and editing. S Thoene: investigation and writing-review and editing. C Winter: investigation and writing-review and editing. R Secci: investigation and writing-review and editing. A Wolf: investigation and writing-review and editing. A Stelzl: investigation and writing-review and editing. K Steiger: investigation and writing-review and editing. J Ruland: investigation and writing-review and editing. F Bassermann: investigation and writing-review and editing. C Kupatt: investigation and writing-review and editing. M Anton: investigation and writing-review and editing. AM Krackhardt: conceptualization, supervision, and writing-review and editing. 


\section{Conflict of Interest Statement}

The authors declare that they have no conflict of interest.

\section{References}

Ahmadi M, King JW, Xue SA, Voisine C, Holler A, Wright GP, Waxman J, Morris E, Stauss HJ (2011) CD3 limits the efficacy of TCR gene therapy in vivo. Blood 118: 3528-3537. doi:10.1182/blood-2011-04-346338

Aurnhammer C, Haase M, Muether N, Hausl M, Rauschhuber C, Huber I, Nitschko H, Busch U, Sing A, Ehrhardt A, et al (2012) Universal real-time PCR for the detection and quantification of adeno-associated virus serotype 2-derived inverted terminal repeat sequences. Hum Gene Ther Methods 23: 18-28. doi:10.1089/hgtb.2011.034

Bassani-Sternberg M, Braunlein E, Klar R, Engleitner T, Sinitcyn P, Audehm S, Straub M, Weber J, Slotta-Huspenina J, Specht K, et al (2016) Direct identification of clinically relevant neoepitopes presented on native human melanoma tissue by mass spectrometry. Nat Commun 7: 13404. doi:10.1038/ncomms13404

Bendle GM, Linnemann C, Hooijkaas Al, Bies L, de Witte MA, Jorritsma A, Kaiser AD, Pouw N, Debets R, Kieback E, et al (2010) Lethal graft-versus-host disease in mouse models of $T$ cell receptor gene therapy. Nat Med 16: 565-570. 561p following 570. doi:10.1038/nm.2128

Clauss J, Obenaus M, Miskey C, Ivics Z, Izsvak Z, Uckert W, Bunse M (2018) Efficient non-viral T-cell engineering by sleeping beauty minicircles diminishing DNA toxicity and miRNAs silencing the endogenous T-cell receptors. Hum Gene Ther 29: 569-584. doi:10.1089/hum.2017.136

ClinicalTrials.gov (2018) NY-ESO-1-redirected CRISPR (TCRendo and PD1) edited T cells (NYCE T cells). 2019.

Cohen CJ, Zhao Y, Zheng Z, Rosenberg SA, Morgan RA (2006) Enhanced antitumor activity of murine-human hybrid T-cell receptor (TCR) in human lymphocytes is associated with improved pairing and TCR/ CD3 stability. Cancer Res 66: 8878-8886. doi:10.1158/0008-5472.can06-1450

Deniger DC, Pasetto A, Tran E, Parkhurst MR, Cohen CJ, Robbins PF, Cooper LJ, Rosenberg SA (2016) Stable, nonviral expression of mutated tumor neoantigen-specific T-cell receptors using the sleeping beauty transposon/transposase system. Mol Ther 24: 1078-1089. doi:10.1038/ mt.2016.51

Doench JG, Hartenian E, Graham DB, Tothova Z, Hegde M, Smith I, Sullender M, Ebert BL, Xavier RJ, Root DE (2014) Rational design of highly active sgRNAs for CRISPR-Cas9-mediated gene inactivation. Nat Biotechnol 32: 1262-1267. doi:10.1038/nbt.3026

Eyquem J, Mansilla-Soto J, Giavridis T, van der Stegen SJ, Hamieh M, Cunanan KM, Odak A, Gonen M, Sadelain M (2017) Targeting a CAR to the TRAC locus with CRISPR/Cas9 enhances tumour rejection. Nature 543: 113-117. doi:10.1038/nature21405

Foley KC, Spear TT, Murray DC, Nagato K, Garrett-Mayer E, Nishimura MI (2017) HCV T cell receptor chain modifications to enhance expression, pairing, and antigen recognition in T cells for adoptive transfer. Mol Ther Oncolytics 5: 105-115. doi:10.1016/j.omto.2017.05.004

Govers C, Sebestyen Z, Coccoris M, Willemsen RA, Debets R (2010) T cell receptor gene therapy: Strategies for optimizing transgenic TCR pairing. Trends Mol Med 16: 77-87. doi:10.1016/j.molmed.2009.12.004

Hacein-Bey-Abina S, Von Kalle C, Schmidt M, McCormack MP, Wulffraat N, Leboulch P, Lim A, Osborne CS, Pawliuk R, Morillon E, et al (2003) LMO2-associated clonal T cell proliferation in two patients after gene therapy for SCID-X1. Science 302: 415-419. doi:10.1126/science.1088547

Hale M, Lee B, Honaker Y, Leung WH, Grier AE, Jacobs HM, Sommer K, Sahni J, Jackson SW, Scharenberg AM, et al (2017) Homology-directed recombination for enhanced engineering of chimeric antigen receptor T cells. Mol Ther Methods Clin Dev 4: 192-203. doi:10.1016/j. omtm.2016.12.008

Harris DT, Kranz DM (2016) Adoptive T cell therapies: A comparison of T cell receptors and chimeric antigen receptors. Trends Pharmacol Sci 37: 220-230. doi:10.1016/j.tips.2015.11.004

Hodgkins A, Farne A, Perera S, Grego T, Parry-Smith DJ, Skarnes WC, Iyer V (2015) WGE: A CRISPR database for genome engineering. Bioinformatics 31: 3078-3080. doi:10.1093/bioinformatics/btv308

June CH, Sadelain M (2018) Chimeric antigen receptor therapy. N Engl J Med 379: 64-73. doi:10.1056/nejmra1706169

Klar R, Schober S, Rami M, Mall S, Merl J, Hauck SM, Ueffing M, Admon A, SlottaHuspenina J, Schwaiger M, et al (2014) Therapeutic targeting of naturally presented myeloperoxidase-derived HLA peptide ligands on myeloid leukemia cells by TCR-transgenic T cells. Leukemia 28: 2355-2366. doi:10.1038/leu.2014.131

Knies D, Klobuch S, Xue SA, Birtel M, Echchannaoui H, Yildiz O, Omokoko T, Guillaume P, Romero P, Stauss H, et al (2016) An optimized single chain TCR scaffold relying on the assembly with the native CD3complex prevents residual mispairing with endogenous TCRs in human T-cells. Oncotarget 7: 21199-21221. doi:10.18632/oncotarget. 8385

Knipping F, Osborn MJ, Petri K, Tolar J, Glimm H, von Kalle C, Schmidt M, Gabriel R (2017) Genome-wide specificity of highly efficient TALENs and CRISPR/Cas9 for T cell receptor modification. Mol Ther Methods Clin Dev 4: 213-224. doi:10.1016/j.omtm.2017.01.005

Krackhardt AM, Anliker B, Hildebrandt M, Bachmann M, Eichmuller SB, Nettelbeck DM, Renner M, Uharek L, Willimsky G, Schmitt M, et al (2018) Clinical translation and regulatory aspects of CAR/TCR-based adoptive cell therapies-the German Cancer Consortium approach. Cancer Immunol Immunother 67: 513-523. doi:10.1007/s00262-0182119-y

Kuball J, Dossett ML, Wolfl M, Ho WY, Voss RH, Fowler C, Greenberg PD (2007) Facilitating matched pairing and expression of TCR chains introduced into human T cells. Blood 109: 2331-2338. doi:10.1182/blood-2006-05023069

Legut M, Dolton G, Mian AA, Ottmann OG, Sewell AK (2018) CRISPR-mediated TCR replacement generates superior anticancer transgenic T cells. Blood 131: 311-322. doi:10.1182/blood-2017-05-787598

Leisegang M, Engels B, Meyerhuber P, Kieback E, Sommermeyer D, Xue SA, Reuss S, Stauss H, Uckert W (2008) Enhanced functionality of T cell receptor-redirected T cells is defined by the transgene cassette. J Mol Med (Berl) 86: 573-583. doi:10.1007/s00109-008-0317-3

Liang X, Weigand LU, Schuster IG, Eppinger E, van der Griendt JC, Schub A, Leisegang M, Sommermeyer D, Anderl F, Han Y, et al (2010) A single TCR alpha-chain with dominant peptide recognition in the allorestricted HER2/neu-specific T cell repertoire. I Immunol 184: 1617-1629. doi:10.4049/jimmunol.0902155

Lim WA, June CH (2017) The principles of engineering immune cells to treat cancer. Cell 168: 724-740. doi:10.1016/j.cell.2017.01.016

MacLeod DT, Antony J, Martin AJ, Moser RJ, Hekele A, Wetzel KJ, Brown AE, Triggiano MA, Hux JA, Pham CD, et al (2017) Integration of a CD19 CAR into the TCR alpha chain locus streamlines production of allogeneic gene-edited CAR T cells. Mol Ther 25: 949-961. doi:10.1016/j.ymthe. 2017.02.005

Mall S, Yusufi N, Wagner R, Klar R, Bianchi H, Steiger K, Straub M, Audehm S, Laitinen I, Aichler M, et al (2016) Immuno-PET imaging of engineered human T cells in tumors. Cancer Res 76: 4113-4123. doi:10.1158/00085472.can-15-2784

Mock U, Hauber I, Fehse B (2016) Digital PCR to assess gene-editing frequencies (GEF-dPCR) mediated by designer nucleases. Nat Protoc 11: 598-615. doi:10.1038/nprot.2016.027

Morgan RA, Dudley ME, Wunderlich JR, Hughes MS, Yang JC, Sherry RM, Royal RE, Topalian SL, Kammula US, Restifo NP, et al (2006) Cancer 
regression in patients after transfer of genetically engineered lymphocytes. Science 314: 126-129. doi:10.1126/science. 1129003

Murnane JP, Yezzi MJ, Young BR (1990) Recombination events during integration of transfected DNA into normal human cells. Nucleic Acids Res 18: 2733-2738. doi:10.1093/nar/18.9.2733

Osborn MJ, Webber BR, Knipping F, Lonetree CL, Tennis N, DeFeo AP, McElroy AN, Starker CG, Lee C, Merkel S, et al (2016) Evaluation of TCR gene editing achieved by TALENS, CRISPR/Cas9, and megaTAL nucleases. Mol Ther 24: 570-581. doi:10.1038/mt.2015.197

Peng PD, Cohen CJ, Yang S, Hsu C, Jones S, Zhao Y, Zheng Z, Rosenberg SA, Morgan RA (2009) Efficient nonviral Sleeping Beauty transposonbased TCR gene transfer to peripheral blood lymphocytes confers antigen-specific antitumor reactivity. Gene Ther 16: 1042-1049. doi:10.1038/gt.2009.54

Provasi E, Genovese P, Lombardo A, Magnani Z, Liu PQ, Reik A, Chu V, Paschon DE, Zhang L, Kuball J, et al (2012) Editing T cell specificity towards leukemia by zinc finger nucleases and lentiviral gene transfer. Nat Med 18: 807-815. doi:10.1038/nm.2700

Rapoport AP, Stadtmauer EA, Binder-Scholl GK, Goloubeva O, Vogl DT, Lacey SF, Badros AZ, Garfall A, Weiss B, Finklestein J, et al (2015) NY-ESO-1specific TCR-engineered T cells mediate sustained antigen-specific antitumor effects in myeloma. Nat Med 21: 914-921. doi:10.1038/ nm.3910

Robbins PF, Morgan RA, Feldman SA, Yang JC, Sherry RM, Dudley ME, Wunderlich JR, Nahvi AV, Helman LJ, Mackall CL, et al (2011) Tumor regression in patients with metastatic synovial cell sarcoma and melanoma using genetically engineered lymphocytes reactive with NY-ESO-1. J Clin Oncol 29: 917-924. doi:10.1200/jco.2010.32.2537

Rosenberg SA, Restifo NP (2015) Adoptive cell transfer as personalized immunotherapy for human cancer. Science 348: 62-68. doi:10.1126/ science.aaa 4967

Roth TL, Puig-Saus C, Yu R, Shifrut E, Carnevale J, Li PJ, Hiatt J, Saco J, Krystofinski P, Li H, et al (2018) Reprogramming human T cell function and specificity with non-viral genome targeting. Nature 559: 405-409. doi:10.1038/s41586-018-0326-5

Scholten KB, Kramer D, Kueter EW, Graf M, Schoedl T, Meijer CJ, Schreurs MW, Hooijberg E (2006) Codon modification of T cell receptors allows enhanced functional expression in transgenic human T cells. Clin Immunol 119: 135-145. doi:10.1016/j.clim.2005.12.009

Schumann K, Lin S, Boyer E, Simeonov DR, Subramaniam M, Gate RE, Haliburton GE, Ye CJ, Bluestone JA, Doudna JA, et al (2015) Generation of knock-in primary human T cells using Cas9 ribonucleoproteins. Proc Natl Acad Sci U S A 112: 10437-10442. doi:10.1073/pnas.1512503112

Seki A, Rutz S (2018) Optimized RNP transfection for highly efficient CRISPR/ Cas9-mediated gene knockout in primary T cells. J Exp Med 215: 985-997. doi:10.1084/jem.20171626

Stronen E, Toebes M, Kelderman S, van Buuren MM, Yang W, van Rooij N, Donia M, Boschen ML, Lund-Johansen F, Olweus J, et al (2016) Targeting of cancer neoantigens with donor-derived $T$ cell receptor repertoires. Science 352: 1337-1341. doi:10.1126/science.aaf2288

Suzuki K, Tsunekawa Y, Hernandez-Benitez R, Wu J, Zhu J, Kim EJ, Hatanaka F Yamamoto M, Araoka T, Li Z, et al (2016) In vivo genome editing via CRISPR/Cas9 mediated homology-independent targeted integration. Nature 540: 144-149. doi:10.1038/nature20565

Tipanee J, VandenDriessche T, Chuah MK (2017) Transposons: Moving forward from preclinical studies to clinical trials. Hum Gene Ther 28: 1087-1104. doi:10.1089/hum.2017.128

Tran E, Robbins PF, Lu YC, Prickett TD, Gartner JJ, Jia L, Pasetto A, Zheng Z, Ray S, Groh EM, et al (2016) T-cell transfer therapy targeting mutant KRAS in cancer. N Engl J Med 375: 2255-2262. doi:10.1056/nejmoa1609279

van Loenen MM, de Boer R, Amir AL, Hagedoorn RS, Volbeda GL, Willemze R, van Rood JJ, Falkenburg JH, Heemskerk MH (2010) Mixed T cell receptor dimers harbor potentially harmful neoreactivity. Proc Natl Acad Sci U S A 107: 10972-10977. doi:10.1073/pnas.1005802107

Zacharakis N, Chinnasamy H, Black M, Xu H, Lu YC, Zheng Z, Pasetto A, Langhan $M$, Shelton $T$, Prickett $T$, et al (2018) Immune recognition of somatic mutations leading to complete durable regression in metastatic breast cancer. Nat Med 24: 724-730. doi:10.1038/s41591018-0040-8

Zhang XH, Tee LY, Wang XG, Huang QS, Yang SH (2015) Off-target effects in CRISPR/Cas9-mediated genome engineering. Mol Ther Nucleic Acids 4: e264. doi:10.1038/mtna.2015.37

Ziegler T, Kraus M, Husada W, Gesenhues F, Jiang Q, Pinkenburg O, Trenkwalder T, Laugwitz KL, le Noble F, Weber C, et al (2017) Steerable induction of the thymosin beta4/MRTF-A pathway via AAV-based overexpression induces therapeutic neovascularization. Hum Gene Ther 29: 1407-1415. doi:10.1089/hum.2017.013

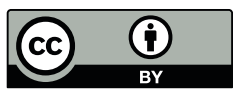

License: This article is available under a Creative Commons License (Attribution 4.0 International, as described at https://creativecommons.org/ licenses/by/4.0/). 